\title{
Autoimmunity overlaps primary biliary cirrhosis: a not straightforward diagnosis
}

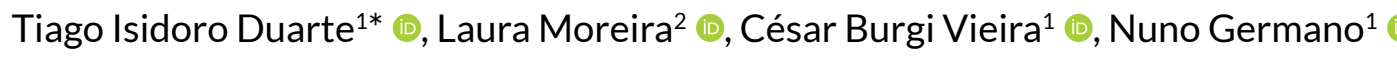

During the last decades, the diagnostic and clinical approach of acute liver failure has been improved due to a progressive understanding of the disease and better outcomes ${ }^{1}$. The most frequent etiologies are viral hepatitis and acetaminophen intoxication, the former in developing countries and the latter in Europe and the United States. Nevertheless, vascular phenomena, infiltrative diseases, or even autoimmune disorders must also be considered ${ }^{2}$. Systemic lupus erythematosus (SLE) is a multisystem autoimmune disorder usually associated with malar rash, arthralgias, cytopenia, serositis, renal failure, endocarditis, and antiphospholipid syndrome ${ }^{3,4}$. Liver involvement is normally not a part of the spectrum but observed in up to $60 \%$ of SLE patients. Moreover, the co-occurrence of autoimmune hepatitis (AIH) and SLE is considered to be rare, and only few reports have been published so far. In this study, we presented a case report of a patient with this overlap syndrome.

A 57-year-old women of Guinean origin presented to the Emergency Department with a 7-day history of jaundice and pruritus. She had a history of arterial hypertension, dyslipidemia, and obesity, treated with lisinopril and pravastatin. She had traveled to a rural area of Guinea-Bissau before 3 months. There was no history of alcohol, drugs, or consumption of natural products. Remarkable physical examination findings included lethargy and flapping without fever. She also had pruritic and papulovesicular skin lesions on torso, upper limbs, and palms but no scalp achievement. Vital signs were normal, and abdominal examination did not reveal pain, tenderness, hepatosplenomegaly, ascites, or lymphadenopathy. Blood tests revealed anemia (hemoglobin $10 \mathrm{~g} / \mathrm{dL}$ ) and thrombocytopenia (platelet account $112 \times 10^{9} / \mathrm{L}$ ), and erythrocyte sedimentation rate of $81 \mathrm{~mm} / \mathrm{h}$. Liver tests showed a cytocholestatic pattern, with a total bilirubin of $20 \mathrm{mg} / \mathrm{dL}$, direct bilirubin of $12 \mathrm{mg} /$ $\mathrm{dL}$, aspartate aminotransferase of $900 \mathrm{IU} / \mathrm{L}$, alanine aminotransferase of $300 \mathrm{IU} / \mathrm{L}$, alkaline phosphatase of $300 \mathrm{IU} / \mathrm{L}$, ammonia of $130 \mu \mathrm{mol} / \mathrm{L}$, hypoalbuminemia of $20.9 \mathrm{~g} / \mathrm{L}$, international normalized ratio of $3: 1$, fibrinogen of $1.04 \mathrm{~g} / \mathrm{L}$, and $\mathrm{V}$ factor of 39\%. Arterial blood gas revealed an elevation in lactate level $(3.0 \mathrm{mmol} / \mathrm{L})$. Acetaminophen levels were negative. Abdominal ultrasound demonstrated a heterogeneous liver with fatty infiltration but without focal lesions or abnormal vascular patterns. Unfortunately, no skin biopsies were performed.

She was admitted to the intensive care department with the diagnosis of acute/subacute liver failure. An extensive workup diagnosis was performed: viral hepatitis $\mathrm{A}, \mathrm{B}, \mathrm{C}$, and $\mathrm{E}$ and herpes simplex serologies were negative. Ceruloplasmin was normal. Coxiella burnetii, rickettsia, brucellosis, schistosomiasis, leptospirosis, and toxoplasmosis were also negative. Polymerase chain reaction (PCR) for Cytomegalovirus (CMV) was positive with viral load of $140 \mathrm{IU} / \mathrm{mL}$ and immunoglobulin $\mathrm{G}$ (IgG) antibodies for the varicella-zoster virus were also positive, so treatment with valganciclovir was initiated. $N$-acetylcysteine protocol was maintained for 5 days and lactulose therapy was performed. According to complementary results, serologies were positive for antinuclear antibodies (homogeneous pattern, 1:640), antidsDNA (773 IU/mL), anti-nucleosome antibodies (>20,000 U/ $\mathrm{mL}$ ), ENA Ro52KD 3+, and hypergammaglobulinemia ( $\mathrm{IgG}+$ ). Serologies were negative for anti-smooth muscle antibody, anti-mitochondrial (AMA), anti-liver kidney microsomal type 1 (LKM 1), anti-cardiolipin, anti-Scl70, and anti-Jo1. C3 and C4 were negative. Transjugular liver biopsy revealed extensive necrosis, portal infiltration by neutrophils, cholestasis, and giant multinuclear cells without steatosis (Figures 1-3). CMV or EpsteinBarr virus (EBV) was not identified by immunohistochemistry. Based on laboratory tests and serologies, a diagnosis of an autoimmune cause was made. Nevertheless, she evolved with bacteremia to Escherichia coli and worsening encephalopathy, so immunosuppressive therapy was postponed, and she underwent liver transplantation at day 7. Postoperative period evolved well with

${ }^{1}$ Centro Hospitalar Universitário de Lisboa Central, Hospital Curry Cabral, Department of Intensive Care Medicine - Lisbon, Portugal.

${ }^{2}$ Centro Hospitalar Universitário de Lisboa Central, Hospital de São José, Department of Internal Medicine - Lisbon, Portugal.

*Corresponding author: tiagomisidoroduarte@gmail.com

Conflicts of interest: the authors declare there is no conflicts of interest. Funding: none.

Received on October 23, 2021. Accepted on December 05, 2021. 


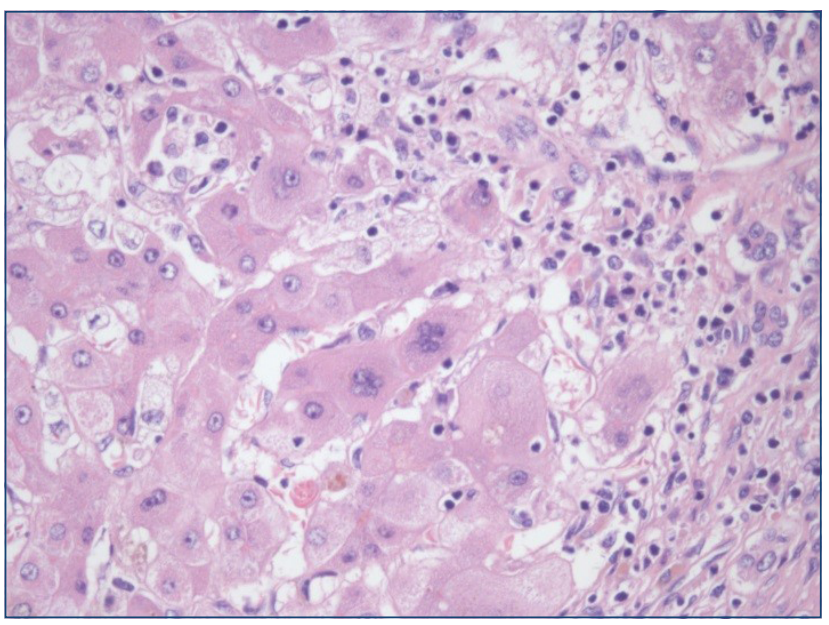

Figure 1. Multinucleated hepatocytes.

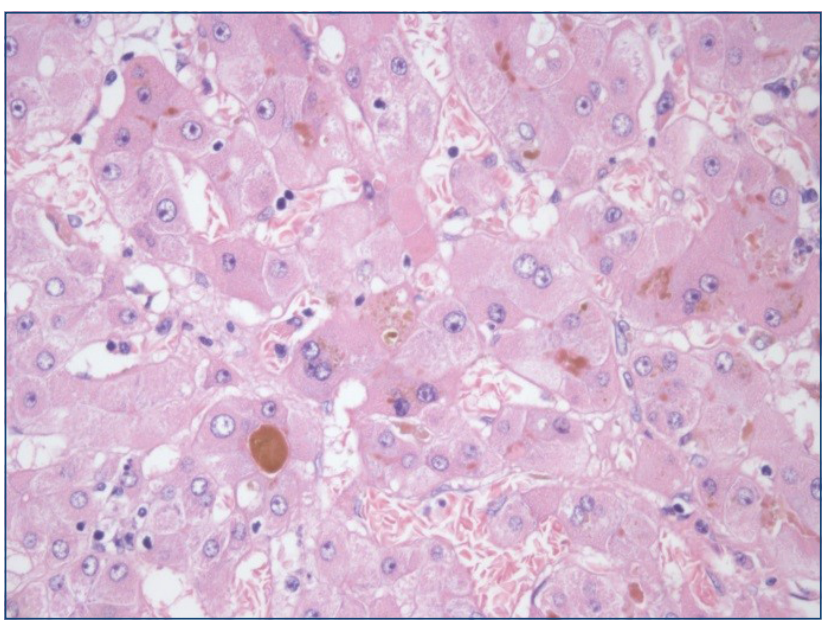

Figure 2. Canalicular and parenchymal cholestasis.

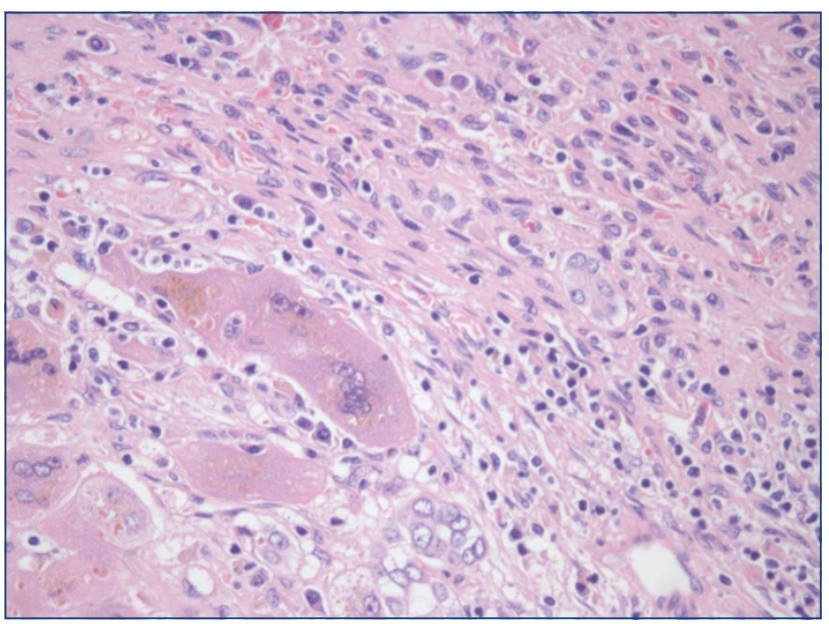

Figure 3. Multinucleated hepatocytes. self-limited cholestasis. Abdominal ultrasound with Doppler was normal. Repeated PCR for CMV was negative. She was discharged after 28 days post-transplant surgery. Immunosuppression therapy was performed first with methylprednisolone and basiliximab and then switched to prednisolone and tacrolimus.

After 3 months, she was again admitted with a progressive liver cytolysis and cholestatic pattern. Abdominal computed tomography revealed a biliary stenosis, and she was then treated with a new biliodigestive derivation. She evolved to hepatic artery thrombosis and subsequent ischemic liver failure and again underwent liver transplantation. Once again, during the postoperative period, she presented a progressive cholestasis under immunosuppression therapy with prednisolone and tacrolimus. Abdominal computed tomography scan was normal. Mycophenolate mofetil was started with progressive regression. Results of 3-month follow-up laboratory liver tests were normal.

SLE is a multisystem autoimmune disorder in which liver involvement is normally not common ${ }^{4}$. AIH, also known as lupoid hepatitis, is an autoimmune liver disease caused by the presence of antibodies (antinuclear antibodies) and hypergammaglobulinemia. The existence of overlap syndromes linking SLE with other autoimmune liver diseases is a matter of controversies, and data in the literature are scarce 5 . Primary biliary cirrhosis $(\mathrm{PBC})$ is a chronic cholestatic disease estimated to evolve to liver failure in $15 \%$ of cases ${ }^{6}$. Besides, AMA antibodies are positive in $95 \%$ of patients with $\mathrm{PBC}$, and clinical presentation, liver histology, and natural history in patients with PBC without AMA antibodies are nearly identical. The study patients are in line with literature as liver biopsy demonstrates the typical features of bile duct destruction and granulomas ${ }^{6}$.

The difference between the hepatic involvement in SLE and AIH has not been clearly defined due to similarities in the clinical and biochemical features ${ }^{7}$. In our report, an unusual presentation associated with a fulminant liver failure course made the diagnostic a challenging task, delaying immunosuppression treatment initiation. A PBC/AIH overlap syndrome may also refer to patients with sequential PBC followed by $\mathrm{AIH}$; less commonly, AIH followed by PBC has been described ${ }^{6}$.

Furthermore, despite liver transplantation, she presented later with new liver cytolysis and cholestatic pattern without any biliary abnormalities under immunosuppression therapy with prednisolone and tacrolimus. According to literature findings, some patients with lupus hepatitis may be refractory not only to corticosteroids but also to conventional immunosuppressants, such as cyclophosphamide, tacrolimus, and azathioprine $^{8}$. As in our case, mycophenolate mofetil led to a rapid resolution of liver test abnormalities. 
In conclusion, AIH and SLE are distinct diseases, in which a combination of clinical symptoms and diagnostics markers overlaps. In our view, AIH needs to be considered in the differential diagnosis of any SLE patient with elevated liver enzymes. Early referencing to a Liver Transplant Centre plays an important key role in these patients' approach and treatment.

\section{ETHICAL APPROVAL}

Our institution does not require ethical approval for reporting individual cases. The patient described herein had given

\section{REFERENCES}

1. Bernal W, Lee WM, Wendon J, Larsen FS, Williams R. Acute liver failure: a curable disease by 2024. J Hepatol. 2015;62(1 Suppl):S112-20. https://doi.org/10.1016/j.jhep.2014.12.016

2. Wendon J, Cordoba J, Dhawan A, Larsen FS, Manns M, Samuel D, et al. Clinical Practice Guidelines: EASL Clinical Practical Guidelines on the management of acute (fulminant) liver failure. J Hepatol. 2017;66(5):1047-81. https://doi.org/10.1016/j.jhep.2016.12.003

3. Cojocaru M,Cojocarul,Silosi I,Vrabie C,Manifestations of systemiclupus erythematosus. Maedica(Buchar).2011;6(4):330-6.PMID: 22879850.

4. Beisel C,Weiler-Normann C, Teufel A, and Lohse AW, et al.Association of autoimmune hepatitis and systemic lupus erythematodes : a consent to the use of de-identified patient data for use in research. Written informed consent was obtained from the patient for this anonymized information to be published in this article.

\section{AUTHORS' CONTRIBUTIONS}

TID: conceptualization, formal analysis, writing - original draft, and writing - review and editing. LM: formal analysis and writing - original draft. CBV: writing - original draft and writing - review and editing. NG: writing - review and editing. case series and review of the literature. World J Gastroenterol. 2014;20(35):12662-7.https://doi.org/10.3748/wjg.v20.i35.12662

5. Bessone F, Poles N, Roma MG. Challenge of liver disease in systemic lupus erythematosus : clues for diagnosis and hints for pathogenesis. World J Hepatol. 2014;6(6):394-409. https://doi. org/10.4254/wjh.v6.i6.394

6. Lindor KD, Gershwin ME, Poupon R, Kaplan M, Bergasa NV, Heathcote EJ. Primary biliary cirrhosis. Hepatology. 2009;50(1):291308. https://doi.org/10.1002/hep.22906

7. Koshy JM, John M, Autoimmune hepatitis-SLE overlap syndrome. J Assoc Physicians India 2012;60:59-60. PMID: 23547416

8. Patel S, Beckler MD, Kesselman MM. Lupus and the Liver : a case study. Cureus. 2019;11(8):1-9.https://doi.org/10.7759/cureus.5477 